\title{
Air-leak Syndrome by Pleuroparenchymal Fibroelastosis after Bone Marrow Transplantation
}

\author{
Tomoya Ishii ${ }^{1}$, Shuji Bandoh ${ }^{1}$, Nobuhiro Kanaji ${ }^{1}$, Akira Tadokoro ${ }^{1}$, Naoki Watanabe ${ }^{1}$, \\ Osamu Imataki ${ }^{1}$, Hiroaki Dobashi ${ }^{1}$, Yoshio Kushida ${ }^{2}$, Reiji Haba ${ }^{2}$ and Hiroyasu Yokomise ${ }^{3}$
}

\begin{abstract}
Objective Air-leak syndrome (ALS) is a life-threatening pulmonary complication following allogeneic bone marrow transplantation (allo-BMT) which is thought to be associated with graft-versus-host disease (GVHD). Recently, it has been reported that pleuroparenchymal fibroelastosis (PPFE) also occurs after allo-BMT and often causes ALS. We sought to extract common features of ALS caused by PPFE after allo-BMT.

Methods The clinical data of patients who developed ALS caused by PPFE after undergoing allo-BMT (ALS-PPFE) between April 1996 and December 2007 at our institution were collected and reviewed retrospectively. The clinical findings, radiological and pathological features and treatment outcomes of ALS-PPFE were assessed.

Results Five patients who developed ALS had histologically proven PPFE (four men, one woman: median age, 37 years). The age of onset of ALS-PPFE was 13 to 109 months (median, 68.8 months) after BMT. Alkylating agents were used as conditioning chemotherapy for BMT in all patients. Only one patient developed chronic GVHD (limited type). The common radiological findings were subpleural thickening and traction bronchiectasis predominantly in the bilateral upper lung fields. The histological pulmonary specimens showed no findings of bronchiolitis obliterans or GVHD. Immunosuppressive therapy was not effective in any of the cases, and all patients died of respiratory failure with or without lung transplantation.

Conclusion ALS-PPFE is an extremely late-onset noninfectious pulmonary complication of allo-BMT. This complication is progressive, resistant to immunosuppressive treatment and has a poor prognosis. No association was found between PPFE and GVHD.
\end{abstract}

Key words: air-leak syndrome, pleuroparenchymal fibroelastosis, allogeneic bone marrow transplantation, late-onset noninfectious pulmonary complications, alkylating agents

(Intern Med 55: 105-111, 2016)

(DOI: 10.2169/internalmedicine.55.4539)

\section{Introduction}

Recent advances in allogeneic bone marrow transplantation (allo-BMT) have improved the prognosis of transplant recipients with hematological diseases. However, pulmonary complications after allo-BMT remain a major cause of mortality and morbidity. Pulmonary complications occur in $25 \%$ to $50 \%$ of patients undergoing allo-BMT, accounting for approximately $50 \%$ of transplant-related deaths (1). The spec- trum of pulmonary complications includes infectious and noninfectious conditions. These conditions are classified as early or late depending on whether they occur before or after 100 days post-transplantation. The term late-onset noninfectious pulmonary complications (LONIPCs) has been used as a generic term for complications occurring later than 100 days after allo-BMT, such as bronchiolitis obliterans (BO), BO organizing pneumonia (BOOP) and interstitial pneumonia (IP)/pulmonary fibrosis (PF). LONIPCs have become recognized as life-threatening complications, and their inci-

\footnotetext{
${ }^{1}$ Department of Internal Medicine, Division of Hematology, Rheumatology and Respiratory Medicine, Faculty of Medicine, Kagawa University, Japan, ${ }^{2}$ Department of Diagnostic Pathology, Faculty of Medicine, Kagawa University, Japan and ${ }^{3}$ Department of General Thoracic, Breast, and Endocrinological Surgery, Faculty of Medicine, Kagawa University, Japan

Received for publication November 18, 2014; Accepted for publication April 26, 2015

Correspondence to Dr. Tomoya Ishii, tishii@ med.kagawa-u.ac.jp
} 
dence has been reported to be $1.7 \%$ to $25.6 \%$ after alloBMT (1). Although the pathogenesis of LONIPCs is unclear, the use of total body irradiation and high-dose chemotherapeutic drugs for conditioning or immunosuppressive drugs for prophylaxis and the treatment of graft-versus-host disease (GVHD) is assumed to be involved.

Air-leak syndrome (ALS), including pneumothorax, pneumomediastinum and subcutaneous emphysema, is a rare complication after allo-BMT (2). ALS usually occurs secondary to $\mathrm{BO}$, which is considered to be a pulmonary manifestation of chronic GVHD in the post-allo-BMT course (2-4). In patients with $\mathrm{BO}$, severe obstruction of the terminal bronchioles can lead to air trapping and bullae development. ALS may be generated by bullae rupture $(3,4)$. Immunosuppressive therapy is a mainstay of treatment for BO after stem cell transplantation, although patients are often resistant to therapy.

Recently, other rare pulmonary complications leading to the development of pneumothorax, pneumomediastinum, subcutaneous emphysema (i.e., ALS) and subpleural parenchymal fibrosis with upper lobe predominance in patients with allo-BMT have been reported (5). These abnormalities are histopathologically consistent with pleuroparenchymal fibroelastosis (PPFE), which was first described as a type of idiopathic interstitial pneumonia by Frankel et al. (5) PPFE is a group of rare forms of idiopathic interstitial pneumonia (IIP) newly classified by the American Thoracic Society/ European Respiratory Society committee in 2013 (6).

We experienced five patients with ALS who developed respiratory failure due to late-onset PPFE after allo-BMT. We herein describe the results of a retrospective analysis of the clinical aspects, histological findings of lung tissues at the time of surgical treatment and outcomes of these patients with ALS caused by PPFE after allo-BMT (ALS-PPFE). In addition, we discuss the pathogenic mechanism of ALSPPFE.

\section{Materials and Methods}

\section{Patients}

We retrospectively analyzed patients with hematologic diseases who received allo-BMT at the Hematology Department of Kagawa University Hospital between April 1996 and December 2007 and survived for at least 100 days after transplantation. Five patients (four men, one woman; median age, 37 years; range, 27-50-years) with ALS-PPFE were identified. Information regarding symptoms, time of onset, radiological and pathological features and treatment outcomes for the patients with ALS-PPFE was collected from the subjects' medical records. Our institution's research ethics board approved this study without the need for subject consent.

\section{Radiological evaluation of ALS}

The diagnosis of ALS was confirmed according to the presence of pneumothorax, pneumomediastinum and subcutaneous emphysema on chest X-ray and computed tomography (CT) scans acquired using an Aquilion CT scanner (Toshiba Medical Systems, Tokyo, Japan). The scans were obtained at end inspiration using 1.0-mm collimation and performed at 5- or 20-mm intervals from the apex of the lung to the diaphragm. High-resolution CT (HRCT) scans were assessed for the presence and extent of ALS and findings definitive of $\mathrm{BO}, \mathrm{BOOP}$ or IP/PF. Mosaic attenuation patterns of the lung parenchyma and patchy consolidations were regarded as signs of $\mathrm{BO}$ and $\mathrm{BOOP}$, respectively. IP/ PF was considered to be present when HRCT demonstrated subpleural thickening, traction bronchiectasis or bronchiolectasis, ground-glass opacification and honeycombing. The HRCT scans were jointly reviewed by three experienced thoracic physicians (T.I., S.B. and N.K.) who recognized the diagnosis of ALS after allo-BMT at the time at which the chest radiographs and CT scans were reviewed. The final decision regarding the findings was made by consensus among all physicians.

\section{Clinicopathological diagnosis of ALS-PPFE}

ALS-PPFE was diagnosed based on the presence of coughing, dyspnea, chest pain and radiological abnormalities 100 days after transplantation without any evidence of an infectious cause. The diagnosis was confirmed according to the histological findings. Because adequate lung material was required to classify the patients as having $\mathrm{BO}, \mathrm{BOOP}$ and/or IP/PF, histological specimens were obtained via video-assisted thoracic surgery (VATS) in four cases and open lung biopsy (OLB) in one case. At least two materials were obtained from the radiologically identified lesions. An autopsy was also performed in one patient with written consent from his family. Tissue cultures were performed to exclude an infectious etiology in all patients. The histological findings were reviewed by two experienced histopathologists (Y.K. and R.H.) at our institution.

\section{Results}

\section{Clinical symptoms and onset}

Five patients survived for at least 100 days after BMT and fulfilled the diagnostic criteria for ALS-PPFE. Their clinical data are shown in Table 1. There were three exsmokers at the time of allo-BMT; the other subjects were never-smokers. No patients had a history of exposure to asbestos or dust or a history of previous pneumothorax or autoimmune disease. ALS-PPFE was diagnosed at a median of 68.8 months after BMT (range, 13-109 months). Three of the five patients showed progressive dyspnea for several months, and two patients developed a sudden onset of chest pain or fever. Unfortunately, pulmonary function tests were not performed at the time of onset because of the presence of air leaks. In addition, all patients did not receive regular yearly checkups, including chest X-ray and pulmonary func- 
Table 1. Clinical Data of the Patients with ALS-PPFE.

\begin{tabular}{|c|c|c|c|c|c|c|c|}
\hline $\begin{array}{l}\text { Case } \\
\text { No. }\end{array}$ & Age/Sex & Smoking status & Symptoms & $\begin{array}{l}\text { Onset day after BMT } \\
\text { (month) }\end{array}$ & $\begin{array}{l}\text { Diagnostic } \\
\text { procedure }\end{array}$ & $\begin{array}{c}\text { Treatment for } \\
\text { pulmonary fibrosis }\end{array}$ & $\begin{array}{l}\text { Survival time from onset } \\
\text { (month) }\end{array}$ \\
\hline \multirow[t]{2}{*}{1} & $44 / F$ & Never & Progressive dyspnea & 39 & OLB & Oral PSL & 13 \\
\hline & & & & & & Bleb/bullectomy & \\
\hline \multirow[t]{4}{*}{2} & $27 / \mathrm{M}$ & Ex-smoker & Sudden fever & 13 & VATS & Oral PSL & 37 \\
\hline & & & & & & Pleurodesis & (alive for 9months \\
\hline & & & & & & Pulmorrhaphy & after lung transplantation) \\
\hline & & & & & & Lung transplantation & \\
\hline \multirow[t]{2}{*}{3} & $38 / \mathrm{M}$ & Ex-smoker & Sudden chest pain, dyspnea & 109 & VATS & Pulmorrhaphy & 16 \\
\hline & & & & & & mPSL pulse & \\
\hline \multirow[t]{3}{*}{4} & $28 / \mathrm{M}$ & Never & Progressive cough, dyspnea & 77 & VATS & Oral CsA & 37 \\
\hline & & & & & Autopsy & Pleurodesis & \\
\hline & & & & & & mPSL pulse & \\
\hline \multirow[t]{2}{*}{5} & $50 / \mathrm{M}$ & Ex-smoker & Progressive dyspnea & 106 & VATS & Oral PSL,CsA & 4 \\
\hline & & & & & & Pleurodesis & \\
\hline
\end{tabular}

OLB: open lung biopsy, VATS: video-assisted thoracic surgery, PSL: prednisolone, mPSL: methyl-prednisolone, CsA : cyclosporine A

Table 2. Conditioning Therapy and Prophylaxis of GVHD.

\begin{tabular}{|c|c|c|c|c|c|c|}
\hline $\begin{array}{l}\text { Case } \\
\text { No. }\end{array}$ & $\begin{array}{l}\text { Underlying } \\
\text { disease }\end{array}$ & $\begin{array}{c}\text { HLA } \\
\text { matching }\end{array}$ & Conditioning & $\begin{array}{c}\text { GVHD } \\
\text { prophylaxis }\end{array}$ & $\begin{array}{l}\text { Acute } \\
\text { GVHD }\end{array}$ & $\begin{array}{l}\text { Chronic } \\
\text { GVHD }\end{array}$ \\
\hline 1 & MDS(RA) & Identical & FL/L-PAM & FK506/PSL & Grade0 & - \\
\hline 2 & MDS(RA) & $1 \mathrm{Ag} \operatorname{mis}(\mathrm{A})$ & FL/L-PAM & FK506/MTX & Grade 0 & - \\
\hline 3 & CML & Identical & $\mathrm{CPA} / \mathrm{TBI}$ & CsA/PSL & Grade 0 & - \\
\hline 4 & ALL & Unknown & $\mathrm{CPA} / \mathrm{BU}$ & CsA/MTX & Grade 0 & - \\
\hline 5 & AA & Identical & CPA/TBI & CsA/MTX & Grade0 & $\begin{array}{c}\text { Limited } \\
\text { (Sicca alone, score1) }\end{array}$ \\
\hline
\end{tabular}

MDS: myelodysplastic syndrome, RA: refractory anemia, CML: chronic myelogenous leukemia,

ALL: acute lymphoid leukemia, AA: aplastic anemia, Ag: antigen, FL: fludarabine,

L-PAM: melphalan, CPA: cyclophosphamide, TBI: total body irradiation, GVHD: graft versus host disease,

PSL: prednisolone, BU: busulfan, CsA: cyclosporin A, MTX: methotrexate, FK506: tacrolimus

tion tests.

\section{Conditioning therapy and prophylaxis}

Three of the five patients with ALS-PPFE had a human leukocyte antigen (HLA)-identical related donor and underwent unrelated BMT (Table 2). Patient 2 had a one-antigen mismatched donor based on human leukocyte antigen typing. As BMT conditioning therapy, all patients were treated with high-dose alkylating agents. Two of the patients received fludarabine $\left(125 \mathrm{mg} / \mathrm{m}^{2}\right)$ and melphalan (140-180 $\left.\mathrm{mg} / \mathrm{m}^{2}\right)$, two received cyclophosphamide (CPA) $(120 \mathrm{mg} / \mathrm{kg}$ ) and total body irradiation (TBI) (12 Gy) and one received CPA and busulfan.

Immunosuppressive agents were given to all patients as GVHD prophylaxis after allo-BMT. Two patients received cyclosporine A (CsA) and methotrexate, one received tacrolimus, methylprednisolone (mPSL) and methotrexate, one received tacrolimus and methotrexate and one received CsA and PSL.

The assessment and grading of acute and chronic GVHD were primarily based on clinical findings and commonly ac- cepted diagnostic criteria $(7,8)$. In particular, chronic GVHD was diagnosed based on its extent and severity according to the clinical scoring system proposed in the National Institutes of Health consensus development project (8). In particular, GVHD was classified as limited (only localized skin and/or liver involvement) or extensive (generalized skin involvement or limited disease plus the involvement of other organs). Only one patient (Patient 5) had limited chronic GVHD and was treated with PSL and CsA. The other patients demonstrated no manifestations of acute or chronic GVHD.

\section{Chest radiography and $\mathrm{CT}$}

All patients with late-onset ALS-PPFE had pneumothorax (Table 3). One patient (Patient 2) exhibited pneumomediastinum and subcutaneous emphysema. Three patients showed bilateral pneumothorax and two had unilateral pneumothorax. The radiological findings were quite similar in all patients. Four patients displayed bilateral subpleural thickening, predominantly in the upper lung fields on chest X-ray (Fig. 1a, c). HRCT findings of the lung parenchyma showed 
Table 3. HRCT Findings of ALS-PPFE.

\begin{tabular}{|c|c|c|c|c|c|c|c|c|c|c|}
\hline $\begin{array}{l}\text { Case } \\
\text { No. }\end{array}$ & Pneumothorax & Pneumomediastinum & $\begin{array}{c}\text { Subcutaneous } \\
\text { emphysema }\end{array}$ & $\begin{array}{c}\text { Traction } \\
\text { bronchiectasis }\end{array}$ & $\begin{array}{l}\text { Subpleural } \\
\text { thickening }\end{array}$ & $\begin{array}{l}\text { Ground-glass } \\
\text { opacification }\end{array}$ & Honeycombing & $\begin{array}{l}\text { Mosaic pattern } \\
\text { of opacification }\end{array}$ & $\begin{array}{c}\text { Patchy } \\
\text { consolidation }\end{array}$ & $\begin{array}{l}\text { Predominance } \\
\text { in upper lobes }\end{array}$ \\
\hline 1 & bilateral & - & - & + & + & + & - & - & - & + \\
\hline 2 & right & + & + & + & - & + & - & - & - & + \\
\hline 3 & right & - & - & + & + & + & - & - & - & + \\
\hline 4 & bilateral & - & - & + & + & + & - & - & - & + \\
\hline 5 & bilateral & - & - & + & + & + & - & - & - & + \\
\hline
\end{tabular}

(a)

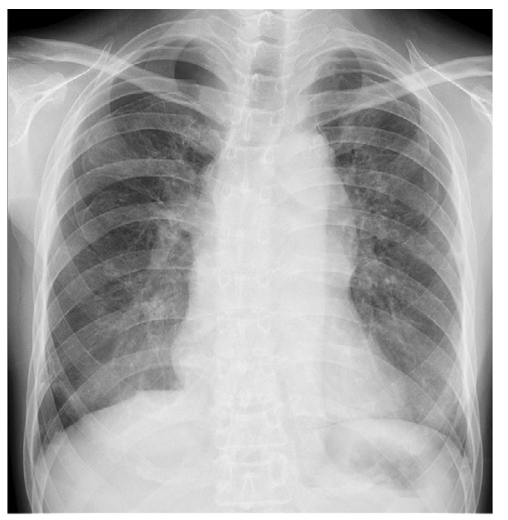

(c)

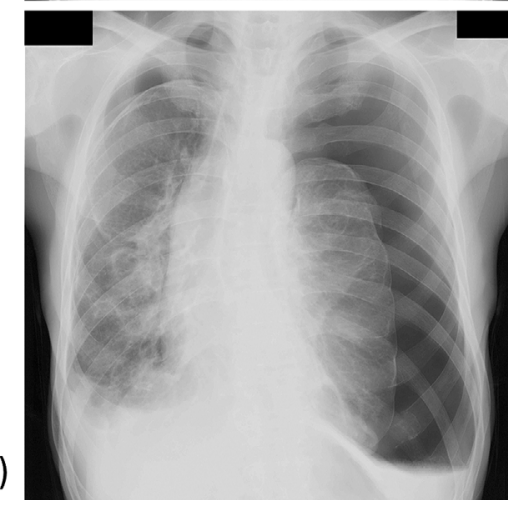

(b)

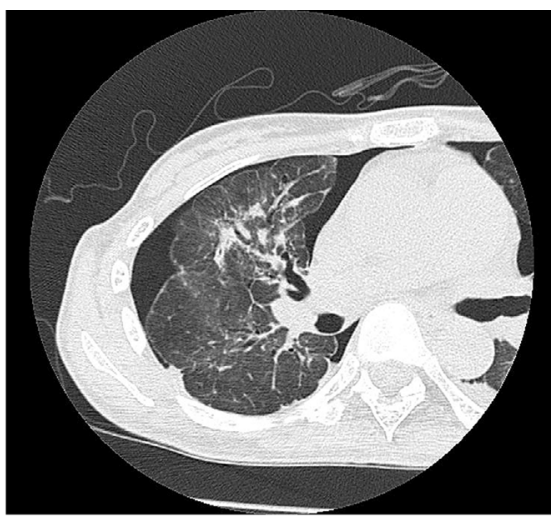

(d)

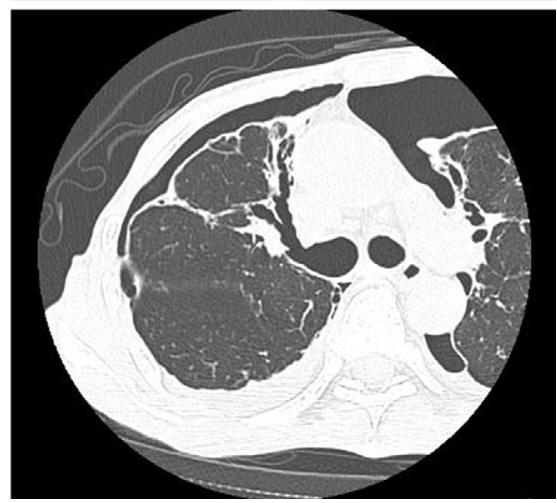

Figure 1. Chest X-ray and HRCT findings of ALS-PPFE. Chest radiographs and HRCT showed subpleural thickening and traction bronchiectasis predominantly found in the bilateral upper lobes with pneumothorax. Patient 1: (a) Chest X-ray and (b) HRCT. Patient 5: (c) Chest X-ray and (d) HRCT.

subpleural thickening, ground-glass opacification along the bronchi and traction bronchiectasis predominantly in the upper lobes (Fig. 1b, d). No honeycombing was observed in any patient (Fig. 1, Table 3), and no signs of expiratory air trapping, bullae formation or patchy consolidation (features of $\mathrm{BO}$ and $\mathrm{BOOP}$ ) were evident in any patient.

\section{Lung biopsies and histological analysis}

The pathological diagnosis of PPFE was determined using VATS in four patients and OLB in one patient. An autopsy was performed in one patient (Patient 4). The pathological findings were quite similar in all patients (Fig. 2). The major pathological findings of the lungs were as follows: (1) multifocal fibrotic lesions involving peribronchial and subpleural lesions accompanied by markedly increased elastic fibers (elastosis) within the alveolar wall, (2) fibrotic lesions predominant in the upper lobes, (3) no significant inflammatory cell infiltration, (4) no honeycombing and (5) no small airway obstruction or organizing pneumonia. Neither epithelioid cell granulomas nor infectious pathogens were observed. These findings were compatible with the diagnosis of PPFE included in the classification of IIP.

The autopsy findings were also pathologically similar. At autopsy, peribronchial and subpleural fibrosis was mainly observed in both upper lobes. A detailed pathological analysis showed no signs of BO or BOOP-associated airway obstruction or bullae formation in the entire lungs, suggesting no histological components of BO and BOOP in ALS-PPFE. 
(a)

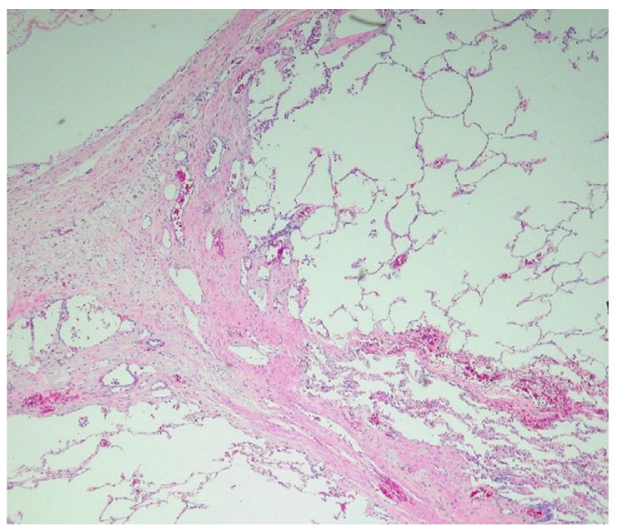

(c)

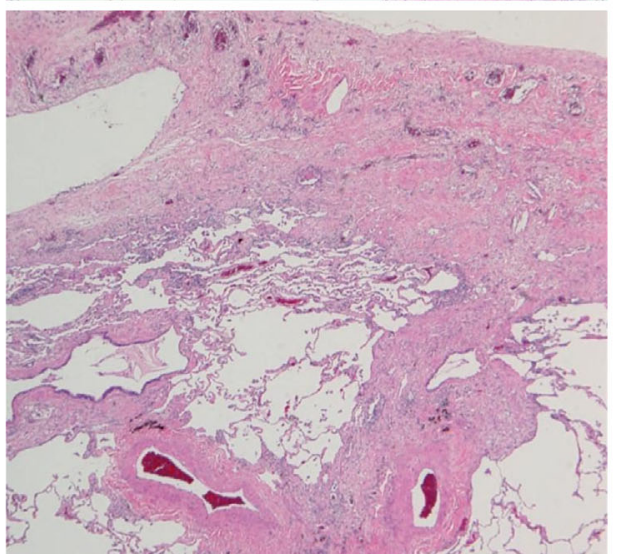

(b)

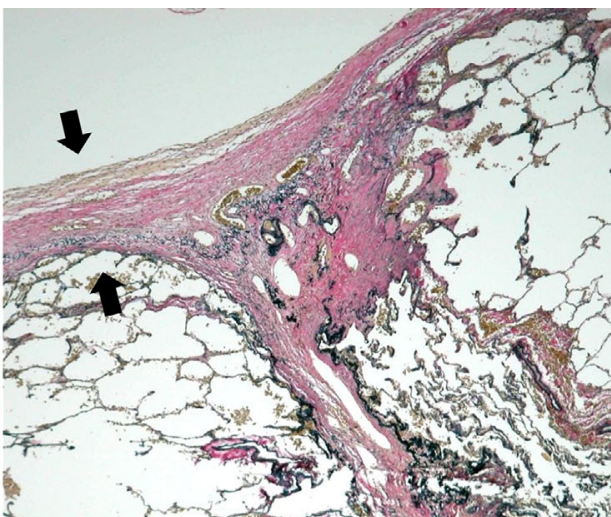

(d)

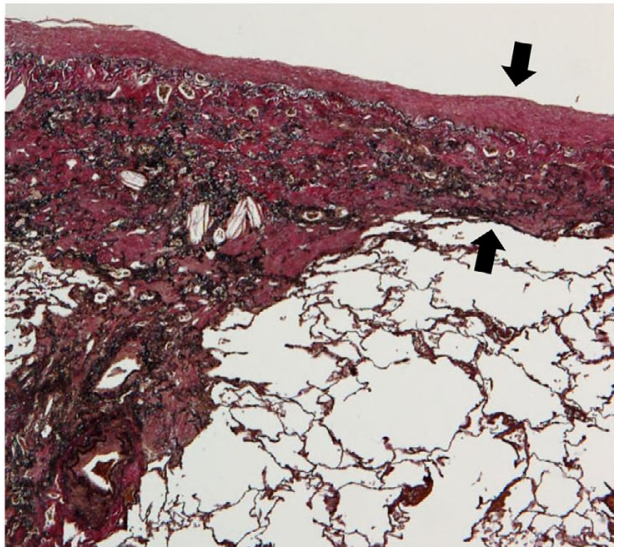

Figure 2. Pathological findings of ALS-PPFE. Pathological findings revealed fibrotic lesions involving peribronchial and subpleural lesions (arrows) accompanied by increased elastic fibers (elastosis) within alveolar walls. Honeycombing was not evident. Patient 1: Right upper lobe (S2). (a) Hematoxylin and Eosin $(H \& E)$ staining $\times 20$, (b) Elastica-Masson stain $\times 20$. Patient 5: Right upper lobe (S2). (c) H\&E staining $\times 20$, (d) Elastica-Masson stain $\times 20$.

\section{Response to therapy and outcomes}

ALS was mainly treated with chest tube placement and oxygen supplementation. All patients received VATS or OLB following pulmorrhaphy and pleurodesis. In addition, all patients were treated with 0.5 to $1.0 \mathrm{mg} / \mathrm{kg} / \mathrm{day}$ of PSL with or without methylprednisolone pulse therapy after the pathological diagnosis of ALS-PPFE. CsA was added in two cases, and the dose of PSL was increased according to the progression of respiratory failure. However, no patients responded to this immunosuppressive therapy. All patients died at a median of 21.4 months after the onset of ALSPPFE (range, 4-37 months). One patient (Patient 2) temporarily recovered from respiratory failure and remained alive for nine months following lung transplantation.

\section{Discussion}

We herein report the results of a retrospective study of the clinicopathological features and outcomes of ALS-PPFE after allo-BMT. The first description of ALS after allo-BMT by Franquet et al. focused on BO and BOOP as the main causes of ALS (3). However, we demonstrated that PPFE can also lead to ALS after allo-BMT. ALS-PPFE may be caused by fibrotic alveolar rupture, leading initially to pul- monary interstitial emphysema and then moving centrally along the bronchovascular sheath into the mediastinum (9).

In this study, ALS-PPFE was diagnosed at a median of 68.8 months after allo-BMT (range, 13-109 months). Common symptoms were progressive dyspnea, coughing and chest pain that appeared suddenly or subacutely just before the diagnosis of ALS. Because most patients with ALSPPFE had no respiratory symptoms for more than five years after allo-BMT, they did not receive regular check-ups involving chest radiographs or pulmonary function tests. On the other hand, several papers have reported ALS-BO/BOOP being diagnosed at a median of 14 months after $\operatorname{BMT}(2,4)$. This difference in the onset time may suggest pathophysiological differences between ALS-PPFE and ALS-BO/ BOOP. From this point of view, the most controversial issue is the role of chronic GVHD in the onset of ALS-PPFE. The histological features of chronic lung GVHD reportedly include active lymphocytic bronchiolitis with epithelial damage and patchy interstitial mononuclear cell infiltration (10-12). Chronic GVHD has been identified as a significant risk factor for $\mathrm{BO} / \mathrm{BOOP}$. Therefore, the risk of ALS-BO/BOOP can be reduced by providing better prevention and control of chronic GVHD. In our hospital, we use a sufficient amount of immunosuppressive agents immediately after allo-BMT for GVHD prevention. In contrast, the role 
of chronic GVHD in the development of ALS-PPFE has not been elucidated. Thüsen et al. speculated that ALS-PPFE is a late complication of GVHD, as pathological BO findings coexisted with PPFE findings in their four patients with ALS-PPFE (13). Fujikura et al. also reported the existence of pathological BO findings in a patient with ALSPPFE (14). Unlike the patients described above, four of our five patients with ALS-PPFE showed no other organ involvement associated with chronic GVHD during the course up to ALS onset. In addition, the histological findings revealed no significant inflammatory cell infiltration within the lung interstitium or peribronchial lesions, such as those observed in BO. These results suggest that our cases of ALS-PPFE were not associated with chronic GVHD. ALSPPFE may occur via a mechanism other than GVHD. We speculate that the reason for the low frequency of chronic GVHD is the use of a sufficient amount of immunosuppressive agents as GVHD prophylactic treatment immediately after allo-BMT in our institution.

It has been reported that both $\mathrm{BO} / \mathrm{BOOP}$ and IP after allo-BMT are caused by GVHD. The first detailed report of IP after allo-BMT was provided by Neiman et al. in 1973, and an association with GVHD was noted (15). In a retrospective review of allo-BMT from the Mayo Clinic, Palmas et al. reported eight patients with unclassifiable IP (4.5\%) among 179 patients during a 14-year period (16). The onset time of IP after BMT was 4 to 54 months (median, 12.8 months). Toubai et al. also reported five cases of ALS caused by IP (ALS-IP) (17). In that report, all patients had chronic GVHD, and the median onset of ALS was seven months after allo-BMT. Furthermore, the radiological features of ALS-PPFE also differ from those of ALS-IP. The chest CT images of ALS-IP reported by Toubai et al. showed bilateral diffuse reticulogranular shadows and ground-glass opacitiy, (17) although subpleural thickening and traction bronchiectasis predominantly occurring in the upper lobes, features of ALS-PPFE, were not described. Yousem classified lung GVHD according to the results of an integrated assessment of the clinical and pathological features of BMT in patients who had undergone OLB (12). In particular, he categorized lung GVHD into four major patterns: 1) diffuse alveolar damage, 2) lymphocytic bronchitis/ bronchiolitis with interstitial pneumonia, 3) BOOP and 4) cicatricial bronchial obstruction. According to this categorization, ALS-IP, as reported by Toubai, is classified into (1) or (2). However, our patients with ALS-PPFE were not classified into such pathological categories. These data suggest that ALS-IP may be induced by chronic GVHD as well as ALS-BO/BOOP and may involve a different pathophysiology to that of ALS-PPFE.

The fundamental question is what factors affect the development of ALS-PPFE. In this regard, the use of alkylating agents and TBI may be associated with the development of ALS-PPFE because these agents have potent PF-inducing effects. In the current study, all patients with ALS-PPFE were treated with alkylating agents, such as CPA, as conditioning therapy for allo-BMT. CPA-induced pulmonary disease is remarkably similar to ALS-PPFE both radiologically and histologically $(18,19)$. Late-onset CPA-induced pulmonary disease (LOCPD) has been reported mostly in patients with Wegener's granulomatosis (granulomatosis with polyangiitis) treated with CPA for long periods (18). The chest X-ray and CT findings noted in these patients consistently demonstrate subpleural thickening and interstitial changes in the bilateral upper lobes. Coughing and dyspnea following ALS often develop after several years of treatment with CPA. The pathological findings of LOCPD reportedly include interstitial deposits of collagen, predominantly in the upper lobes, with small amounts of inflammatory cells, such as lymphocytes and plasma cells. Honeycombing and other features of IIP are not observed. The pathological findings of carmustine (BCNU)-induced late-onset lung disease in bone marrow transplant patients are also similar to those of LOCPD (20). Based on the above evidence, the administration of alkylating agents may be a proposed cause of ALSPPFE. TBI also has potent PF-inducing effects. However, three of our five patients with ALS-PF did not receive TBI, suggesting that TBI is not required for the development of ALS-PPFE.

ALS-PPFE is an important cause of post-transplantation morbidity and mortality in addition to ALS-BO/BOOP. None of our patients with ALS-PPFE responded to immunosuppressive therapy, and all patients died at a median of 21.4 months after the onset of ALS-PPFE (range, 4-37 months). One patient temporarily recovered from respiratory failure and remained alive for nine months after receiving lung transplantation. Our findings highlight the irreversible nature of this complication despite the use of conventional immunosuppressive therapy. Providing the proper management of ALS-PPFE is especially challenging, and the development of new therapeutic modalities, such as living donor lung transplantation, would help to decrease post-BMT morbidity and mortality.

In summary, ALS-PPFE is an extremely late-onset noninfectious pulmonary complication after BMT. The clinical symptoms of ALS-PPFE may be insidious at the beginning of the disease process, and this condition may have no association with GVHD. Moreover, the application of alkylating agents may be a proposed cause of ALS-PPFE. This complication is progressive, resistant to immunosuppressive treatment as well as pulmonary transplantation and has a poor prognosis. It would also be valuable to undertake a prospective analysis of late-onset lung complications following alloBMT.

\section{The authors state that they have no Conflict of Interest (COI).}

\section{References}

1. Yoshihara S, Yanik G, Cooke KR, Mineishi S. Bronchiolitis obliterans syndrome (BOS), bronchiolitis obliterans organizing pneumonia (BOOP), and other late-onset noninfectious pulmonary 
complications following allogeneic hematopoietic stem cell transplantation. Biol Blood Marrow Transplant 13: 749-759, 2007.

2. Kumar S, Tefferi A. Spontaneous pneumomediastinum and subcutaneous emphysema complicating bronchiolitis obliterans after allogeneic bone marrow transplantation: case report and review of literature. Ann Hematol 80: 430-435, 2001.

3. Franquet T, Rodríguez S, Hernández JM, et al. Air-leak syndromes in hematopoietic stem cell transplant recipients with chronic GVHD: high-resolution CT findings. J Thorac Imaging 22: 335340, 2007.

4. Moon MH, Sa YJ, Cho KD, Jo KH, Lee SH, Sim SB. Thoracic air-leak syndromes in hematopoietic stem cell transplant recipients with graft-versus-host disease: a possible sign for poor response to treatment and poor prognosis. J Korean Med Sci 25: 658-662, 2010.

5. Frankel SK, Cool CD, Lynch DA, Brown KK. Idiopathic pleuroparenchymal fibroelastosis: description of a novel clinicopathologic entity. Chest 126: 2007-2013, 2004.

6. Travis WD, Costabel U, Hansell DM, et al; ATS/ERS Committee on Idiopathic Interstitial Pneumonias. An official American Thoracic Society/European Respiratory Society statement: Update of the international multidisciplinary classification of the idiopathic interstitial pneumonias. Am J Respir Crit Care Med 188: 733-748, 2013.

7. Przepiorka D, Weisdorf D, Martin P, et al. 1994 Consensus Conference on Acute GVHD Grading. Bone Marrow Transplant 15: 825-828, 1995.

8. Filipovich AH, Weisdorf D, Pavletic S, et al. National Institutes of Health consensus development project on criteria for clinical trials in chronic graft-versus-host disease: I. Diagnosis and staging working group report. Biol Blood Marrow Transplant 11: 945-956, 2005.

9. Macklin CC. Transport of air along sheats of pulmonic blood vesels from alveoli to mediastinum. Clinical implications. Arch Intern Med 64: 913-926, 1939.

10. Beschorner WE, Saral R, Hutchins GM, Tutschka PJ, Santos GW.
Lymphocytic bronchitis associated with graft-versus-host disease in recipients of bone-marrow transplants. $N$ Engl J Med 299: 1030-1036, 1978.

11. Sloane JP, Depledge MH, Powles RL, Morgenstern GR, Trickey BS, Dady PJ. Histopathology of the lung after bone marrow transplantation. J Clin Pathol 36: 546-554, 1983.

12. Yousem SA. The histological spectrum of pulmonary graft-versushost disease in bone marrow transplant recipients. Hum Pathol 26: 668-675, 1995.

13. von der Thüsen JH, Hansell DM, Tominaga M, et al. Pleuroparenchymal fibroelastosis in patients with pulmonary disease secondary to bone marrow transplantation. Mod Pathol 24: 1633-1639, 2011.

14. Fujikura Y, Kanoh S, Kouzaki Y, Hara Y, Matsubara O, Kawana A. Pleuroparenchymal fibroelastosis as a series of airway complications associated with chronic graft-versus-host disease following allogeneic bone marrow transplantation. Intern Med 53: 43-46, 2014.

15. Neiman $P$, Wasserman $P B$, Wentworth $B B$, et al. Interstitial pneumonia and cytomegalovirus infection as complications of human marrow transplantation. Transplantation 15: 478-485, 1973.

16. Palmas A, Tefferi A, Myers JL, et al. Late-onset noninfectious pulmonary complications after allogeneic bone marrow transplantation. Br J Haematol 100: 680-687, 1998.

17. Toubai T, Tanaka J, Kobayashi N, et al. Mediastinal emphysema and bilateral pneumothoraces with chronic GVHD in patients after allogeneic stem cell transplantation. Bone Marrow Transplant 33: 1159-1163, 2004.

18. Malik SW, Myers JL, DeRemee RA, Specks U. Lung toxicity associated with cyclophosphamide use. Two distinct patterns. Am J Respir Crit Care Med 154: 1851-1856, 1996.

19. Hamada K, Nagai S, Kitaichi M, et al. Cyclophosphamide-induced late-onset lung disease. Intern Med 42: 82-87, 2003.

20. Parish JM, Muhm JR, Leslie KO. Upper lobe pulmonary fibrosis associated with high-dose chemotherapy containing $\mathrm{BCNU}$ for bone marrow transplantation. Mayo Clin Proc 78: 630-634, 2003.

(C) 2016 The Japanese Society of Internal Medicine http://www.naika.or.jp/imonline/index.html 